\title{
Nachwort des Apostolischen Nuntius bei der Schweizerischen Eidgenossenschaft und im Fürstentum Liechtenstein, Erzbischof Thomas E. Gullickson
}

Abgesehen von meiner echten Leidenschaft für die Geschichte der Kirche im Allgemeinen berührt das Thema der Nuntiatur in Luzern die historischen Wurzeln meines gegenwärtigen Arbeitsfeldes hier in Bern und damit gewissermassen meine Identität als päpstlicher Vertreter in der Schweizerischen Eidgenossenschaft und im Fürstentum Liechtenstein. Es bot mir die Gelegenheit, einen Vergleich zwischen den beiden Nuntiaturen anzustellen, oder, wenn Sie möchten, zwischen einer Vertretung mit einem klaren Profil aus der Gegenreformation und jener, deren jetziger Titular ich bin und deren Profil ich nicht genauer definieren könnte.

Das Erscheinen der Dissertation von Mario Galgano im Jahr 2020 fällt mit dem 450. Jahrestag der Reise des hl. Karl Borromäus in die Schweiz im August 1570 zusammen. Diese gab den Anstoss zur Errichtung der Apostolischen Nuntiatur mit Sitz in Luzern, die seit dem Jahr 1586 zur festen Einrichtung wurde.

Die Dissertation bietet einen differenzierten Überblick über die verschiedenen Gründe dieser borromäischen Initiative und über die Tätigkeit der päpstlichen Vertreter, die in der ersten Periode des Bestehens der Nuntiatur in Luzern dienten. Wie Galgano zeigt, gehen die Aufgaben der päpstlichen Mission in Luzern weit über die übliche humorvolle Bemerkung hinaus, nach der die kirchlichen Adligen es in fast 300 Jahren Aufenthalt hier auf Schweizer Gebiet versäumt haben, den Bischof von Konstanz dazu zu zwingen, Residenz zu halten und die Reformen des Konzils von Trient umzusetzen.

$\mathrm{Zu}$ Beginn meines Aufenthalts hier in der Schweiz hatte ich das Glück, das wegweisende Werk von Urban Fink lesen zu können: Die Luzerner Nuntiatur 1586-1873. ${ }^{1}$ Galganos These trägt zu einer wachsenden Zahl von Monografien bei, die Fink als Ausgangspunkt nehmen und unser Wissen über diese Periode und ihre Protagonisten erweitern. Legitim oder nicht, ich sehe in Galganos Werk auch ein offenes Fenster auf die Zeitgeschichte der Kirche in der Schweiz. Die in seiner Doktorthese angegebenen Quellen sprechen nicht nur von den

1 Urban Fink: Die Luzerner Nuntiatur 1586-1873.

Hinweis: Erzbischof Thomas E. Gullickson war von 2015 bis 2020 Apostolischer Nuntius in der Schweiz.

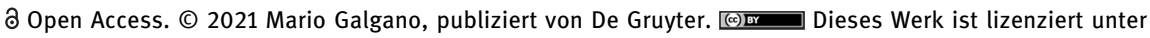
der Creative Commons Attribution 4.0 Lizenz. Weitere Informationen finden Sie unter 
Nuntien und der Nuntiatur jener Zeit, sondern bieten uns einen Vergleich zwischen den katholischen Gemeinschaften jener Zeit und den heutigen mit all ihren Verdiensten und Problemen, Gaben und Mängeln.

Galgano gelingt es auch, eine doppelte Besonderheit der „Reformnuntiatur“ in Luzern zu beschreiben. Trotz des Wunsches der katholischen Kantone, eine päpstliche Gesandtschaft zu errichten, beruhte ihre Errichtung nicht auf der gegenseitigen Entsendung von Botschaftern zwischen Rom und der Eidgenossenschaft. Darüber hinaus war die Nuntiatur in Luzern, vielleicht wie nirgendwo sonst auf der Welt, an der Rekrutierung von Söldnern beteiligt (was heute in der Päpstlichen Schweizergarde weiterlebt), um die Person des Papstes zu verteidigen und, für eine gewisse Zeit, als Soldaten für den Kriegseinsatz und die politische Förderung des Kirchenstaates Dienst zu leisten.

Beim Lesen der Zusammenfassungen des Briefwechsels der einzelnen Nuntien dieser Zeit verspüre ich einen gewissen Neid auf die Klarheit der Aufgaben, die ihnen in der Zeit der Gegenreformation gestellt wurden. Trotz der Ähnlichkeiten zwischen unseren Missionen muss man sagen, dass ein Nuntius des 21. Jahrhunderts mit einem Profil leben muss, das weniger klar umschrieben ist. Nuntius Ludovico di Sarego (1613-1621) z. B. konnte beim französischen Botschafter an seine katholische Identität appellieren und ihn auffordern, seine Pflicht gegenüber dem Heiligen Vater und der Schweiz im Konflikt mit Venedig $\mathrm{zu}$ erfüllen. Ich sage dies, obwohl ich dankbar bin, dass wir uns nicht mit Kriegsfragen befassen müssen, geschweige denn mit der Rekrutierung und Bezahlung von Söldnern als Kämpfer bei kleinen Kriegsausbrüchen hier und dort auf der Welt.

Ein weiterer grosser Unterschied zwischen der Ausübung des Amtes in Luzern vor 400 Jahren und meiner Arbeit heute wäre die Natürlichkeit, mit der sich die Nuntien der damaligen Zeit in Angelegenheiten des Amtes untereinander verständigten. Die Nuntien fungieren heute ausschliesslich als Vermittler zwischen dem Heiligen Stuhl und dem einzelnen Akkreditierungsland mit seiner Ortskirche. Es wäre interessant, den Grund und das Datum des gegenwärtigen Entscheides zu kennen, das den päpstlichen Vertretern verbietet, in Angelegenheiten von gemeinsamem Interesse direkt mit ihren anderen NuntiusKollegen zu korrespondieren - ganz im Gegensatz zu ihren diplomatischen Laien-Kollegen.

Überraschend, aber angesichts des Profils einer Reformnuntiatur auch verständlich, ist das Interesse des Nuntius Carlo Carafa della Spina (1653-1654) an der Frage der Berufungskrise in Teilen seines Hoheitsgebiets. Er schreibt deutlich über den Mangel an Priestern, um das sakramentale Leben zugunsten der katholischen Gläubigen zu sichern. Bei allen Nuntien dieser Zeit denkt man an 
ihre Aufgabe, die sie durch das Konzil von Trient erhalten hatten, nämlich die Einrichtung von Seminaren zu fördern:

\begin{abstract}
Sie werden mit allen möglichen Überlegungen die Gründung von Priesterseminaren ins Auge fassen, indem sie die Bischöfe und Ordenskapitel auffordern, die Befehle des Heiligen Konzils von Trient umzusetzen. Dies ist im Übrigen zu ihrem eigenen Vorteil. Vor allem hegt der Bischof von Sitten den großen Wunsch hegt, ein Seminar in seinem Bistum zu errichten, deshalb werden Sie sich mit dem Bischof und dem Klerus austauschen und sie davon überzeugen, so viel wie möglich zu tun, um dieses so heilige Werk zu vollbringen, was dem Allgemeinwohl sehr nützlich ist. ${ }^{2}$
\end{abstract}

Unter anderem fehlt bei keinem der Nuntien der ersten Periode die Überzeugung, dass der Glaube nur bei konfessioneller Homogenität erhalten werden kann. Es scheint auch, dass die protestantischen Behörden zu dieser Zeit bestrebt waren, katholische Minderheiten aus ihren Kantonen zu vertreiben. ${ }^{3}$ Die Schwierigkeiten der katholischen Enklaven, den katholischen Glauben zu bewahren und unnötige Konflikte zwischen den Konfessionen zu vermeiden, werden viel diskutiert. Obwohl inzwischen Jahrhunderte vergangen sind und das Zweite Vatikanische Konzil mit seiner Lehre über die Ökumene stattgefunden hat, beanspruchen einige Kantone vielleicht aus diesem Grund immer noch eine gewisse Kompetenz als Garanten des religiösen Friedens.

Die Städte Freiburg und Bern teilen sich vier Untertanengebiete, die sie gemeinsam führen. Die Gefahr besteht darin, dass sich die dortigen Katholiken durch den Handel mit den Häretikern mit der Häresie infizieren könnten. Deshalb braucht Euer Hochwohlgeboren alle Informationen über dieses Geschäft vom Bischof von Veglia. Da müssen Sie Ihren Beitrag dazu leisten, wenn die Herren von Freiburg Sie darum bitten. Sie erhalten zu Ihren Händen ein entsprechendes Breve über diese Anordnung, die in Kopien anderen Schriften beigefügt und die man Ihnen zur Kenntnisnahme über weitere Einzelheiten geben wird. ${ }^{4}$

In diesen Anweisungen von Kardinal Borghese an den Nuntius Verallo wird für das Personal der Nuntiatur eine typische Bezeichnung jener Zeit verwendet: „Familie“.

Mir scheint nun, Ihnen einige wenige Dinge noch mitteilen zu müssen, die mit Ihrem Gefolge zu tun haben, auch wenn ich denke, dass dies der Qualität entspricht, die einer Person zukommt, damit sie auch anderen als Beispiel dienen kann. Ich will Sie daran erinnern, dass Sie Menschen mitbringen sollten, die wohlerzogen sind, fromm und ruhig, und dass die zwei wichtigsten Mitarbeiter, der Auditor und der Sekretär, intelligent sein müs-

2 Siehe Seite 196 im vorliegenden Band.

3 Diese Methode wurde auch auf katholischer Seite so gehandhabt, z. B. im Bistum Sitten galt es, entweder wieder katholisch zu werden oder den Kanton zu verlassen.

4 Ebd. 
sen, und Sie müssen sie darauf aufmerksam machen, dass sie bei ihrer Tätigkeit gewissenhaft handeln, und sie an die Würde dieses Heiligen Stuhls erinnern, der nicht undankbar ist gegenüber jenen, die ihm gut dienen. ${ }^{5}$

Einerseits erklärt diese Bezeichnung bestimmte Aspekte der Verwaltung päpstlicher Vertretungen auch im 21. Jahrhundert, wie z. B. das Rechnungsführungssystem fast ohne Kontrollen, das heisst, es liegt praktisch immer noch im Ermessen des Leiters der Vertretung. Ich frage mich, ob eine Rückkehr zum Begriff „Familie“ nicht eine Möglichkeit wäre, die Krise bei der Personalrekrutierung für die Nuntiaturen zu lösen, indem es jedem Nuntius überlassen bleibt, seinen eigenen „Auditor“ und „Sekretär“ zu finden. Vielleicht war es falsch, die Staaten bei der Pflege einer diplomatischen Karriere zu imitieren? Ich muss sagen, dass ich die Präzision und Offenheit der Beschreibungen genoss, die nicht nur Kardinal Borghese, sondern auch die einzelnen Nuntien jener Zeit über die Schweiz verfassten.

Es gibt viele Kantone, viele Republiken, und alle haben dementsprechend in ihren Ländern die höchste Autorität inne. Nicht zuletzt wird ein Teil von ihnen aristokratisch und demokratisch regiert. Aristokraten regieren in Zürich, Bern, Luzern, Solothurn, Freiburg und Schaffhausen. ${ }^{6}$

Vom Volk regiert sind alle anderen, nämlich Altdorf, Schwyz, Unterwalden, Zug, Appenzell und Glarus. Die Anführer der aristokratischen Republiken werden an einigen Orten Bürgermeister genannt, und diese befinden sich in Zürich, Basel und Schaffhausen. An einigen anderen Orten wird diese Autorität Schultheiß genannt, wie in Luzern, Freiburg und Solothurn. Unter den demokratischen Kantonen werden sie Ammann oder Landammann, das heißt Beamter des Landes, genannt. Aber alle haben die gleiche Autorität, fast wie der Doge von Venedig oder die Konsuln im antiken Rom. ${ }^{7}$

In demselben Bericht bemerkt der Nuntius über den schweizerischen Teil der Diözese Konstanz:

In Helvetien weiß ich nicht, ob durch Privileg oder alten Brauch alle Pfarreien und [nicht leserlich] von den gleichen Herren der Republiken versorgt werden oder, wie in einigen Kantonen wie Schwyz und Appenzell, bis hin zu den Pfarrern von den Landsleuten bedroht und vertrieben werden können, wenn sie mit einem Priester unzufrieden sind, was sie auch tatsächlich tun. Daher ist der arme Priester manchmal gezwungen, Unwürdigkeiten $\mathrm{zu}$ begehen, um geliebt und bestätigt zu werden, und aus dieser Quelle geht hervor, dass es in diesen Gebieten keine guten Untertanen gibt, die einem dienen wollen, aber der Mangel ist zwar bedauerlich, jedoch nicht heilbar.“

5 Siehe Seite 200 im vorliegenden Band.

6 Siehe Seite $215 \mathrm{im}$ vorliegenden Band.

7 Siehe Seite 215 im vorliegenden Band. Vermutlich 1620. Bericht über die Nuntiatur in der Schweiz von Ladislao d'Aquino, Bischof von Venafro. 
„Bei dieser Gelegenheit ist über dieses Thema zu sagen, dass es das Gerücht gibt, in Helvetien gebe es Simonie, und das ist wahrscheinlich wegen der Gier der weltlichen Herrscher, die keinen ordentlichen Priester bevorzugen, ohne an ihre Interessen zu denken. ${ }^{8}$

Vielleicht wird der Name „Babylonische Gefangenschaft“, der der katholischen Kirche in der Schweiz in unserer Zeit zugeordnet wird, hier und nicht durch die Mängel des gegenwärtigen dualen Systems erklärt. Wir sind es gewohnt, die Mängel des dualen Systems auf die Auferlegung eines Modells zurückzuführen, das besser für die Protestanten geeignet ist, doch stattdessen scheint es einen Teil der Schweizer Mentalität, auch der katholischen, widerzuspiegeln - ganz im Gegensatz zu der des deutschen Teils von Konstanz.

Ich wünsche dem Autor, dass seine Dissertation einen Platz einnehme im facettenreichen historischen Mosaik und uns dahinführe, die schweizerisch-katholische Realität mit wohlwollendem Blick besser zu verstehen. Die Reformnuntiatur in Luzern spielte eine zentrale Rolle bei den Bemühungen in der Zeit nach dem Konzil von Trient, die katholische Kirche zu reformieren und ihre Position und damit ihren Dienst für die Kirche in Europa als leuchtende Stadt auf dem Berg zu stärken.

Bern, 12. Dezember 2019

+ Thomas E. Gullickson

Titularerzbischof von Bomarzo

Apostolischer Nuntius bei der Schweizerischen Eidgenossenschaft und im Fürstentum Liechtenstein

8 Siehe Seite $237 \mathrm{im}$ vorliegenden Band. 
